\title{
Dietary availability in elderly Portuguese households
}

\author{
Débora M Santos ${ }^{1,2, *}$, Sara SP Rodrigues ${ }^{1,3}$, Bruno MPM Oliveira ${ }^{1,4}$ \\ and Maria Daniel $V$ de Almeida \\ 'Faculdade de Ciências da Nutrição e Alimentação da Universidade do Porto (FCNAUP), Rua Dr. Roberto Frias, \\ 4200-465 Porto, Portugal: ${ }^{2}$ Department of Social Nutrition, Institute of Nutrition, Rio de Janeiro State University, Rio \\ de Janeiro, Brazil: ${ }^{3}$ Instituto de Saúde Pública da Universidade do Porto (ISPUP), Porto, Portugal: ${ }^{4}$ Laboratório de \\ Inteligência Artificial e Apoio à Decisão do Instituto de Engenharia de Sistemas e Computadores do Porto (LIAAD - \\ INESC), Porto, Portugal
}

Submitted 3 September 2012: Final revision received 24 January 2014: Accepted 27 February 2014: First published online 24 April 2014

\begin{abstract}
Objective: To identify dietary availability and its time trends in elderly Portuguese households.

Design: A set of four cross-sectional studies based on the Household Budget Surveys was used. The dietary data were described using the daily per capita availability of food and beverages, energy and selected nutrients (macronutrients, different lipid fractions and simple sugars). Differences between elderly household types and time trends were studied.

Setting: Portuguese Household Budget Survey data from 1989/1990, 1994/1995, 2000/2001 and 2005/2006.

Subjects: Households with members aged $\geq 65$ years were selected and categorized as solitary elderly female, solitary elderly male or couple (composed of one elderly female and one elderly male).

Results: While cereals, fats/oils, potatoes and sugar/sugar products decreased, an increase occurred in milk/milk products, fruits, bottled water, fruit/vegetable juices and soft drinks $(P<0.05)$. The highest values for foods and beverages were mostly found in couples, while the lowest ones were from solitary males. Exceptions were observed for cereals, eggs, milk/milk products, vegetables, fruits and non-alcoholic beverages, higher in solitary females; and for sugar/sugar products and alcoholic beverages, higher in solitary males. Over time, total energy and carbohydrates decreased while proteins and saturated fatty acids increased $(P<0.001)$. Lipids increased in solitary males and couples $(P<0.05)$. Simple sugars increased in solitary males but decreased in solitary females and couples $(P<0 \cdot 05)$.

Conclusions: The increases in fruits and vegetables in solitary females accord with a healthier food pattern, but overall imbalances in the macronutrient profile for all elderly households may imply a decreasing diet quality.
\end{abstract}

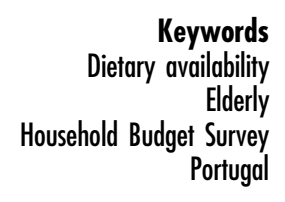

Throughout the world, populations are ageing ${ }^{(1)}$. In 2010, people aged 65 years and over constituted $17.4 \%$ of the total population in Europe, a proportion that is expected to continue to increase in the coming decades ${ }^{(1,2)}$. The Portuguese population faces a similar trend. Demographic projections for Portugal estimate that in 2050, the elderly will represent $32 \%$ of the entire population ${ }^{(3)}$.

This unprecedented situation has prompted researchers to study the relationships between modifiable factors, namely physical activity and diet, because these factors are related to the development of chronic diseases and healthy ageing ${ }^{(4-6)}$. Due to its potential to modulate the transition from vulnerability to frailty and dependence, nutrition is an important determinant of the quality of ageing ${ }^{(6,7)}$. Examination and monitoring of the dietary characteristics of the elderly is important in identifying subgroups at risk of malnutrition or disease ${ }^{(8-10)}$. Given the need to incorporate a life course perspective ${ }^{(11)}$ into public health nutrition initiatives, identification of the dietary habits of elderly individuals contributes to the development of more specific and practical nutrition programmes and directed interventions.

At the country level, national health surveys, food balance sheets and household budget surveys are the main sources of data that provide direct and indirect information on dietary intake. In Portugal, national studies evaluating 
direct dietary intake data are scarce ${ }^{(12-16)}$. Several papers have used indirect dietary data from the Household Budget Surveys (HBS) ${ }^{(17,18)}$ to examine household food availability and diet quality in Portugal, including their characteristics and time trends ${ }^{(19-23)}$. Using the same approach, the association between different localities, household diet quality and mortality data has also been studied $^{(23,24)}$.

Although the HBS are not designed for dietary assessment, a comparison of food and nutrient availability based on the results of the HBS with dietary data values measured at different levels of dietary intake assessment present similar estimates ${ }^{(25,26)}$. Furthermore, HBS are collected periodically and allow the study of the effect of socio-economic and demographic characteristics on daily food choices ${ }^{(20)}$.

Although dietary characterization has previously been examined in Portugal, existing data on this topic are limited. If we consider the elderly population, the available information is even scarcer and more fragmented ${ }^{(27,28)}$. Food availability has been reported for elderly people from European countries, including Portugal, in the DAFNE-ANEMOS Project ${ }^{(20)}$. Diet quality has also been studied for European elderly households ${ }^{(29)}$ and for Portuguese elders. ${ }^{(28)}$ However, only one study has specified the household availability of food, energy and nutrients for older adults, and it focused on Portuguese elderly individuals living alone in the year $2000^{(30)}$.

Therefore, the purpose of the present study was to identify dietary availability and its time trends in elderly Portuguese households with respect to food and beverage groups, energy and selected nutrients.

\section{Methodology}

\section{Study design and participants}

The present study used the last four available editions of the Portuguese HBS, which cover representative samples of the population. The National Institute of Statistics is responsible for these cross-sectional surveys, which included 12403 households in 1989/1990, 10554 households in 1994/1995, 10020 households in 2000/2001 and 10403 households in 2005/2006. The sampling was performed through a multiple-stage technique. Primary units were the second level of the Nomenclature of Units for Territorial Statistics (NUTS 2) and secondary units were the households in each area, according to census data ${ }^{(31-34)}$.

The households included in the present analysis were composed exclusively by elders ( $\geq 65$ years) ${ }^{(35)}$. They were categorized according to sex, and number of members, as follows: 'solitary elderly female' households; 'solitary elderly male' households; and 'couple' households, composed of one elderly female and one elderly male. Due to the small sample size of other types of households (less than $3.9 \%$ of the total households with elders) we did not include households with more than two elderly (with very different compositions), households with two males and households with two females. Our sample comprised 1967 households in 1989/1990, 2219 households in 1994/1995, 2533 households in 2000/2001 and 2441 households in 2005/2006.

\section{Sociodemographic variables}

Sociodemographic characteristics were considered in terms of several variables. The educational level of the household head was categorized as 'illiterate/elementary' for no formal education to 6 years of formal education, 'secondary' from 9 to 12 years of formal education and 'higher', indicating a bachelor's degree, master's degree or PhD. The household locality urbanization degree was described as 'rural', 'semi-urban' or 'urban'. The locations of the households in Portuguese regions were defined according to NUTS 2: 'North', 'Central', 'Lisbon and Tejo Valley', 'Alentejo', 'Algarve', 'Azores' and 'Madeira'. Economic welfare was identified by the 'per capita income' (Euro/person per d); the 'total food expenses', expressed as a proportion of total household expenditure (\%); and the 'eating out expenses', expressed as a proportion of total food expenses (\%).

\section{Dietary estimates}

The HBS food and beverage acquisition information (in terms of both quantity and monetary value) were obtained through a self-registration diary of the items entering the household (purchased, home produced or received as gifts) during $7 \mathrm{~d}$ in 1989/1990 and during $14 \mathrm{~d}$ in the 1994/1995 version onwards. The data were collected during a 12-month period to capture seasonal variability. Detailed methodological information about the surveys is described elsewhere ${ }^{(31-34)}$.

The household food availability data were recorded in approximately 500 different codes and categorized into food and beverages groups according to the DAFNE (Data Food Networking) procedures ${ }^{(17)}$ and its food classification scheme $^{(18)}$. A slight deviation from this classification was introduced: 'fruit/vegetable juices' were described separately as well as under the main group of 'non-alcoholic bev-

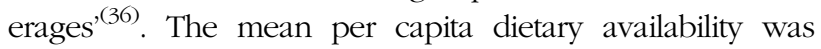
estimated through the division of the household daily availability by the household size. The fourteen main food groups used were described as follows: 'cereals' (cereal and cereal products), 'meat/meat products' (meat, meat products and dishes), 'fish/seafood', 'eggs', 'milk/milk products', 'added fats/oils', 'potatoes' (potatoes and other starchy roots), 'pulses', 'nuts', 'vegetables', 'fruits', 'sugar/sugar products', 'nonalcoholic beverages' (stimulants, i.e. coffee, tea and cocoa; bottled water; fruit/vegetable juices; soft drinks) and 'alcoholic beverages' (wine, beer and spirits).

The average daily energy (MJ/person per $\mathrm{d}$ ) and nutrient availability (in weight or proportion of total energy available at the household level), namely 'proteins', 'lipids', 'lipid 
fractions' (MUFA, SFA and PUFA), 'cholesterol', 'carbohydrates' and 'simple sugars', were estimated. The software MicrodietPlus for Windows version 1·1 2000, which is based on McCance and Widdowson's food composition table and its updated supplements adapted to include Portuguese foods ${ }^{(37)}$, was used.

\section{Statistical methods}

Weights were used to take into account the sample design effect. Descriptive statistics included means, standard errors, medians, quartiles and frequencies. All continuous variables had a positively skewed distribution. In order to reduce skewness they were log transformed. To study time trends of (the log of) income, food expenses and eating out expenses, as well as for the (the log of) quantities of each food and beverage group, we used the two-way ANOVA with effects of time, household type and their interaction. Contrasts were used to investigate whether each analysed variable increased with time. The effect size was interpreted using the value of the partial $\eta^{2}$ through a qualitative definition of betweensubject effects proposed by Cohen ${ }^{(38)}$. This criterion identifies the overall adjusted importance of the analysed variables. The interpretation should be taken as follows: when we say that there is a large effect size for a food and beverage group it means that the evaluated sociodemographic variables and time have a great influence on this food and beverage group mean household availability. Most of the effect sizes were small $\left(\eta^{2}<0.035\right)$; medium $\left(0 \cdot 035 \leq \eta^{2}<0 \cdot 100\right)$ and large $\left(\eta^{2} \geq 0 \cdot 100\right)$ effect sizes ${ }^{(38)}$ are denoted in the text when applicable. The significances and effect sizes presented in the tables were obtained from the log-transformed variables and we chose to show the means and their standard errors from the original data (before applying the $\log$ ). To estimate the effect of time (Y) and household type $(\mathrm{HH})$ in each categorical variable (V; educational level of the household head, urbanization and region) we used a log-linear multinomial model: $\mathrm{V}+\mathrm{HH}+$ $\mathrm{Y}+(\mathrm{HH} \times \mathrm{V})+(\mathrm{Y} \times \mathrm{V})+(\mathrm{Y} \times \mathrm{HH})$. All reported $P$ values were two-sided and were considered statistically significant at the $0 \cdot 05$ level. The statistical analysis was performed in IBM SPSS Statistics version 20.0.

\section{Results}

The participants' sociodemographic characteristics are shown in Table 1 . The most frequent elderly household types were those composed of couples and solitary female elderly individuals. The majority of the elderly households were headed by individuals with an illiterate/elementary level of education. Approximately half of the elderly households were located in urban areas and this proportion increased over time, with a decrease in households in rural areas. Elderly households were concentrated mainly in the Lisbon and Tejo Valley, North and Central regions.
The household per capita income was highest in the solitary male elderly household type $(P<0 \cdot 001)$ and, over time, it increased for all elderly household types (large effect size, $P<0.001)$ at different rates $(P<0.001)$. The total food expenses impacted the budget for all elderly household types, but for solitary male elderly households, it presented the highest percentages $(P<0 \cdot 001)$. Overall, the proportion of total food expenses decreased over time (medium effect size, $P<0.001$ ) but at different rates $(P<0.001)$ in each household. In contrast, eating out expenses for all elderly household types were low and increased over time $(P<0.001)$ at different rates $(P<0.001)$. The solitary elderly male households had the highest eating out expenses $(P<0 \cdot 001)$.

Table 2 presents the daily per capita availability of food and beverage groups among the elderly Portuguese household types according to survey year. Differences among household types were found for all food and beverage groups $(P \leq 0 \cdot 003)$. Medium effect sizes were present for alcoholic beverages and wine (with highest availability within the solitary elderly males), meat/meat products (with highest availability within the couple households) and vegetables (with lowest availability within the solitary elderly males). Among all household types, there was a significant variation over time $(P \leq 0.044)$ in the availability of all food and beverage groups except nuts. The largest, albeit small, effect sizes were found for cereals (decreased), milk/milk products (increased) and potatoes (decreased). Furthermore, we observed an increase in fruits, bottled water, fruit/vegetable juices and soft drinks, and a decrease in added fats/ oils and sugar/sugar products. Distinctions in the variations over time between households were rather small but significant $(P \leq 0.044)$ for all food and beverage groups except for: eggs, added fats/oils, beer and fruits.

The median values of per capita daily total dietary energy and nutrients available for elderly household types by survey year are presented in Table 3. Differences among household types had higher, but small, effect sizes in cholesterol (lower values in solitary elderly males), simple sugars (higher values in solitary elderly females) and MUFA (higher in the couple households). From 1990 to 2005 , the largest, even if small, variations were found for SFA and proteins (both increased) and for energy (decreased). Distinctions in the variations over time between households were quite small but significant $(P \leq 0 \cdot 001)$ for energy and all nutrients except for: PUFA and carbohydrates.

\section{Discussion}

The current study is the first to present detailed information about dietary availability in elderly Portuguese households over a 15 -year period. Attention was paid to the description and the direction of the changes in dietary 
Table 1 Sociodemographic characteristics of elderly Portuguese households. Household Budget Surveys from 1990 to 2005

\begin{tabular}{|c|c|c|c|c|c|c|c|c|c|c|c|c|c|c|c|c|c|c|c|c|c|c|c|c|}
\hline \multirow[b]{4}{*}{ Sociodemographic characteristic } & \multirow{2}{*}{\multicolumn{12}{|c|}{ Household type }} & \multicolumn{12}{|c|}{ Effects $\dagger$} \\
\hline & & & & & & & & & & & & & & & & & & & & & Interac & ction terms & & \\
\hline & \multicolumn{4}{|c|}{ Solitary elderly female } & \multicolumn{4}{|c|}{ Solitary elderly male } & \multicolumn{4}{|c|}{ Couple* } & \multicolumn{2}{|c|}{ Variable $(\mathrm{V})$} & \multicolumn{2}{|c|}{ Household type $(\mathrm{HH})$} & \multicolumn{2}{|c|}{ Year $(Y)$} & \multicolumn{2}{|c|}{$\mathrm{HH} \times \mathrm{V}$} & \multicolumn{2}{|c|}{$Y \times V$} & \multicolumn{2}{|r|}{$\mathrm{Y} \times \mathrm{HH}$} \\
\hline & 1990 & 1995 & 2000 & 2005 & 1990 & 1995 & 2000 & 2005 & 1990 & 1995 & 2000 & 2005 & $\chi_{\mathrm{df}}^{2}$ & $P$ & $\chi^{2} \mathrm{df}$ & $P$ & $x^{2} \mathrm{df}$ & $P$ & $\chi^{2} \mathrm{df}$ & $P$ & $\chi_{\mathrm{df}}^{2}$ & $P$ & $\chi^{2} \mathrm{df}$ & $P$ \\
\hline $\begin{array}{l}\text { Sample size } \\
\text { Education of household head } \\
(\%)\end{array}$ & 744 & 824 & 1027 & 913 & 204 & 242 & 296 & 281 & 1019 & 1153 & 121 & 1247 & & & & & & & & & & & & \\
\hline $\begin{array}{l}\text { Illiterate/elementary } \\
\text { Secondary }\end{array}$ & $\begin{array}{r}96.7 \\
2.2\end{array}$ & $\begin{array}{r}92.4 \\
5.2\end{array}$ & $\begin{array}{r}91.0 \\
5.0\end{array}$ & $\begin{array}{r}90.9 \\
6.6\end{array}$ & $\begin{array}{r}91 \cdot 8 \\
6 \cdot 3\end{array}$ & $\begin{array}{r}93 \cdot 3 \\
3.1\end{array}$ & $\begin{array}{r}95 \cdot 0 \\
3.8\end{array}$ & $\begin{array}{r}88.0 \\
7.2\end{array}$ & $\begin{array}{r}92.1 \\
6.0\end{array}$ & $\begin{array}{r}91.8 \\
6.8\end{array}$ & $\begin{array}{r}87.8 \\
9.0\end{array}$ & $\begin{array}{r}89.3 \\
7.4\end{array}$ & $19177 \cdot 1_{12}$ & $<0.001$ & $7555 \cdot 4_{12}$ & $<0.001$ & $127 \cdot 8_{15}$ & $<0.001$ & $30.7_{4}$ & $<0.001$ & $53 \cdot 86$ & $<0.001$ & $37 \cdot 2_{6}$ & $<0.001$ \\
\hline \multirow{2}{*}{\multicolumn{25}{|c|}{$\begin{array}{l}\text { Locality urbanization degree } \\
(\%)\end{array}$}} \\
\hline & & & & & & & & & & & & & & & & & & & & & & & & \\
\hline $\begin{array}{l}\text { Urban } \\
\text { Semi-urban }\end{array}$ & $\begin{array}{l}48 \cdot 8 \\
18 \cdot 3\end{array}$ & $\begin{array}{l}54.8 \\
22.9\end{array}$ & $\begin{array}{l}48.0 \\
25.8\end{array}$ & $\begin{array}{l}66.1 \\
12.6\end{array}$ & $\begin{array}{l}42 \cdot 3 \\
23 \cdot 6\end{array}$ & $\begin{array}{l}37 \cdot 1 \\
32 \cdot 1\end{array}$ & $\begin{array}{l}49 \cdot 4 \\
26 \cdot 6\end{array}$ & $\begin{array}{l}58 \cdot 8 \\
18.0\end{array}$ & $\begin{array}{l}45 \cdot 3 \\
20 \cdot 4\end{array}$ & $\begin{array}{l}44 \cdot 3 \\
27.4\end{array}$ & $\begin{array}{l}44.8 \\
26.7\end{array}$ & $\begin{array}{l}60.5 \\
14.0\end{array}$ & $1941 \cdot 7_{12}$ & $<0.001$ & $7563 \cdot 2_{12}$ & $<0.001$ & $403 \cdot 3_{15}$ & $<0.001$ & $38.5_{4}$ & $<0.001$ & $329 \cdot 3_{6}$ & $<0.001$ & $35 \cdot 5_{6}$ & $<0.001$ \\
\hline $\begin{array}{l}\text { Semm-urban } \\
\text { Rural }\end{array}$ & 32.9 & $22 \cdot 3$ & $\begin{array}{l}25 \cdot 8 \\
26 \cdot 3\end{array}$ & $\begin{array}{l}12.6 \\
21 \cdot 3\end{array}$ & $\begin{array}{l}23.6 \\
34.1\end{array}$ & $32 \cdot 1$ & $\begin{array}{l}26 \cdot 6 \\
24 \cdot 1\end{array}$ & $23 \cdot 2$ & $\begin{array}{l}20 \cdot 4 \\
34 \cdot 3\end{array}$ & $\begin{array}{l}28.4 \\
28.3\end{array}$ & $\begin{array}{l}28.1 \\
28.5\end{array}$ & $\begin{array}{l}14.0 \\
25.5\end{array}$ & & & & & & & & & & & & \\
\hline \multicolumn{25}{|l|}{ Portuguese regions (\%) } \\
\hline North & $28 \cdot 1$ & $27 \cdot 2$ & $26 \cdot 3$ & 28.6 & 32.5 & $24 \cdot 6$ & $24 \cdot 5$ & $26 \cdot 4$ & $27 \cdot 0$ & 30.4 & 29.4 & 27.5 & $7734 \cdot 1_{30}$ & $<0.001$ & $5181 \cdot 7_{18}$ & $<0.001$ & $96 \cdot 6_{24}$ & $<0.001$ & $19 \cdot 9_{10}$ & $<0.001$ & $71 \cdot 9_{15}$ & $<0.001$ & $18.9_{6}$ & $<0.001$ \\
\hline Central & 20.4 & 18.3 & 21.2 & 24.8 & 23.9 & 28.6 & $16 \cdot 2$ & 26.4 & 21.4 & 21.2 & 20.8 & 29.0 & & & & & & & & & (1) & & & \\
\hline Lisbon and Tejo Valley & 32.6 & 40.1 & 38.0 & 29.2 & 27.8 & 29.5 & 37.8 & 29.2 & $33 \cdot 3$ & 34.2 & 34.5 & 24.6 & & & & & & & & & & & & \\
\hline Alentejo & 8.7 & $6 \cdot 1$ & 7.1 & 10.4 & 6.2 & $10 \cdot 3$ & $10 \cdot 4$ & 9.6 & 9.4 & 7.3 & 7.8 & 11.9 & & & & & & & & & & & & \\
\hline Algarve & 6.1 & 4.0 & 4.3 & 4.4 & $6 \cdot 2$ & 4.0 & 8.3 & 6.4 & $6 \cdot 2$ & 4.4 & 4.9 & 4.8 & & & & & & & & & & & & \\
\hline \multicolumn{25}{|l|}{ Autonomous regions } \\
\hline \multirow{3}{*}{$\begin{array}{l}\text { Azores } \\
\text { Madeira }\end{array}$} & $\begin{array}{l}2.0 \\
2.0\end{array}$ & $\begin{array}{l}1.6 \\
2.7\end{array}$ & $\begin{array}{l}1.5 \\
1.8\end{array}$ & $\begin{array}{l}1.4 \\
1.3\end{array}$ & $\begin{array}{l}1.9 \\
1.4\end{array}$ & $\begin{array}{l}1.8 \\
1.3\end{array}$ & $\begin{array}{l}1.2 \\
1.7\end{array}$ & $\begin{array}{l}1.2 \\
0.8\end{array}$ & $\begin{array}{l}1.5 \\
1.2\end{array}$ & $\begin{array}{l}1.5 \\
1.0\end{array}$ & $\begin{array}{l}1.2 \\
1.3\end{array}$ & $\begin{array}{l}1.1 \\
1.0\end{array}$ & & & & & & & & & & & & \\
\hline & \multicolumn{12}{|c|}{ Household type } & & & Effec & & & & & & & & & \\
\hline & Solit & itary elc & derly fen & male & & litary el & derly $\mathrm{m}$ & & & $\mathrm{Cou}$ & uple* & & Househ & hold type & $\mathrm{Ye}$ & & Intera & action§ & & & & & & \\
\hline Sociodemographic characteristic & 1990 & 1995 & 2000 & 2005 & 1990 & 1995 & 2000 & 2005 & 1990 & 1995 & 2000 & 2005 & $\eta^{2}$ & $P$ & $\eta^{2}$ & $P$ & $\eta^{2}$ & $P$ & & & & & & \\
\hline Per capita income (Euros/d) & & & & & & & & & & & & & & & & & & & & & & & & \\
\hline Mean & 4.9 & $12 \cdot 2$ & 12.6 & 23.6 & 7.1 & $13 \cdot 0$ & $15 \cdot 7$ & $32 \cdot 1$ & 4.9 & 9.7 & 11.8 & 20.9 & 0.018 & $<0.001$ & 0.270 & $<0.001$ & 0.003 & $<0.001$ & & & & & & \\
\hline SE & 0.1 & 0.4 & 0.4 & 0.6 & 0.6 & 0.9 & 0.9 & 1.9 & 0.1 & 0.2 & 0.2 & 0.4 & & & & & & & & & & & & \\
\hline P25 & 2.8 & $6 \cdot 2$ & 6.6 & 13.8 & 3.1 & 6.0 & 7.5 & $16 \cdot 7$ & 2.7 & 5.4 & 6.1 & $12 \cdot 0$ & & & & & & & & & & & & \\
\hline Median & 4.2 & 8.9 & 9.8 & 18.7 & 4.5 & 8.7 & $10 \cdot 1$ & 23.9 & 3.6 & 7.4 & $8 \cdot 1$ & $15 \cdot 9$ & & & & & & & & & & & & \\
\hline P75 & $6 \cdot 2$ & $13 \cdot 3$ & $12 \cdot 7$ & 26.8 & 7.5 & 14.0 & $19 \cdot 1$ & $35 \cdot 0$ & 5.7 & 11.2 & $12 \cdot 7$ & 23.8 & & & & & & & & & & & & \\
\hline $\begin{array}{l}\text { Total food expenses (\% of total } \\
\text { household expenses) }\end{array}$ & & & & & & & & & & & & & & & & & & & & & & & & \\
\hline $\begin{array}{l}\text { nousenold expenses) } \\
\text { Mean }\end{array}$ & 52.9 & 40.2 & 36.4 & 29.1 & 56.4 & 50.5 & $44 \cdot 3$ & $35 \cdot 3$ & $51 \cdot 6$ & $43 \cdot 3$ & 39.2 & 30.6 & 0.007 & $<0.001$ & 0.054 & $<0.001$ & 0.003 & $<0.001$ & & & & & & \\
\hline SE & 0.7 & 0.7 & 0.6 & 0.6 & 1.4 & 1.6 & 1.4 & 1.1 & 0.4 & 0.4 & 0.4 & 0.3 & & & & & & & & & & & & \\
\hline P25 & 39.7 & 24.0 & 21.3 & 16.4 & $42 \cdot 1$ & $30 \cdot 7$ & $29 \cdot 1$ & $22 \cdot 4$ & 37.8 & 29.4 & 25.8 & $20 \cdot 3$ & & & & & & & & & & & & \\
\hline Median & 52.4 & 38.8 & 33.7 & $26 \cdot 1$ & $56 \cdot 3$ & 51.9 & $48 \cdot 1$ & 33.3 & 52.5 & 42.7 & 36.9 & 28.3 & & & & & & & & & & & & \\
\hline P75 & $67 \cdot 2$ & 54.4 & 48.3 & 39.0 & 72.7 & 69.8 & 59.5 & $47 \cdot 1$ & 64.6 & 55.7 & 50.1 & 38.7 & & & & & & & & & & & & \\
\hline Eating out expenses (\% of total & & & & & & & & & & & & & & & & & & & & & & & & \\
\hline $\begin{array}{l}\text { food expenses) } \\
\text { Mean }\end{array}$ & 16.8 & 14.1 & 17.0 & 22.3 & $27 \cdot 8$ & 23.4 & 38.2 & 48.8 & 7.7 & 9.2 & 11.7 & $15 \cdot 2$ & 0.023 & $<0.001$ & 0.016 & $<0.001$ & 0.004 & $<0.001$ & & & & & & \\
\hline SE & 1.0 & 0.9 & 0.9 & 1.1 & 2.3 & 2.2 & 2.2 & 2.4 & 0.3 & 0.4 & 0.4 & 0.5 & & & & & & & & & & & & \\
\hline P25 & 0.0 & 0.0 & 0.0 & 0.0 & 0.0 & 0.0 & 4.8 & 10.3 & 0.0 & 0.0 & 0.0 & 0.0 & & & & & & & & & & & & \\
\hline Median & 0.0 & 0.0 & 0.0 & 2.9 & 11.6 & $2 \cdot 1$ & 30.1 & 54.2 & 0.0 & 0.0 & 0.7 & 4.8 & & & & & & & & & & & & \\
\hline P75 & 29.4 & 18.8 & 22.4 & 37.6 & 54.0 & 43.7 & 68.9 & 83.3 & 6.7 & 11.6 & 14.4 & 23.0 & & & & & & & & & & & & \\
\hline
\end{tabular}

P25, 25th percentile; P75, 75th percentile; $\eta^{2}$, partial eta squared.

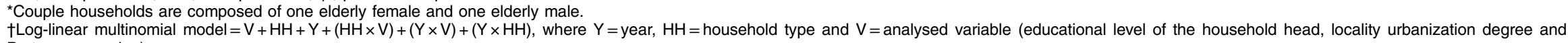
Portuguese region).

$\ddagger$ Two-way ANOVA with effects of time, household type and their interaction was used to test for time trends.

§Interaction $=$ household type $\times$ year. 
Table 2 Daily per capita food and beverage availability in elderly Portuguese households. Household Budget Surveys from 1990 to 2005

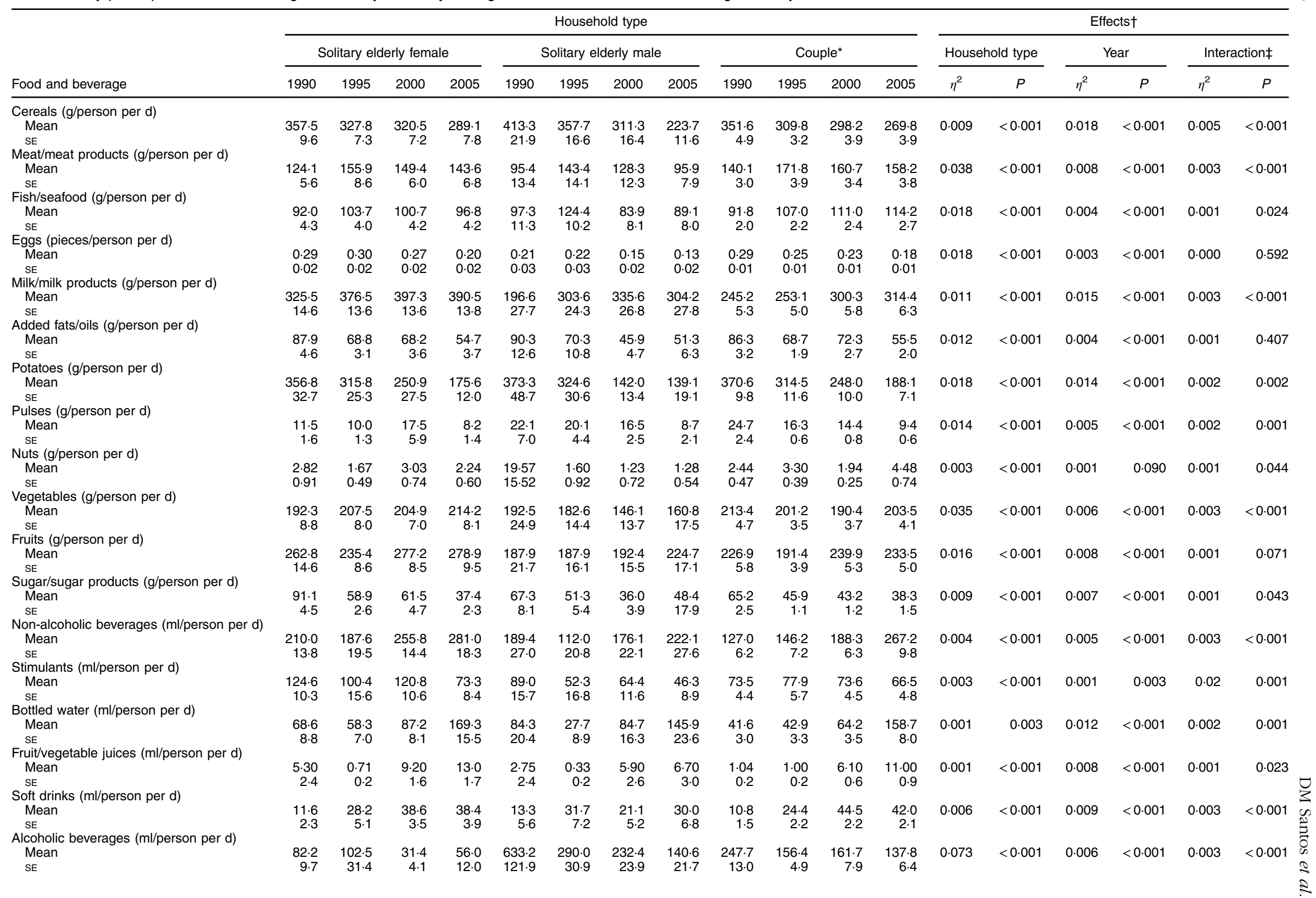


availability within households with different elderly compositions, including those with a solitary elderly female, a solitary elderly male and couple elderly households. Our findings will be compared with the available national household dietary information and with data from other European countries. Furthermore, data from other Portuguese studies describing dietary information will be used, especially data focused on elderly individuals.

The more evident changes over time in the food and beverage availability within the elderly Portuguese households were a reduction of cereals and potatoes and an increase of milk/milk products. These changes within the elderly households are in line with those observed for the entire Portuguese population ${ }^{(19,36,39)}$. However, data from the last edition of the National Health Survey ${ }^{(16)}$ on direct measures of intake revealed that cereals, such as rice, pasta and bread, as well as potatoes were products that were present in approximately $90 \%$ of Portuguese meals. Although elderly households may be more traditional than other households in their household food availability, results may indicate a transition from the traditional diet to a more 'modern' one. In particular, the increase over time of milk/milk products, but also of bottled water, fruit/vegetable juices and soft drinks may exemplify the "modernization" of dietary availability because these products became more readily available in the late $1990 \mathrm{~s}^{(16,40)}$. An increasing pattern for soft drinks over the years was also found in European countries, where their availability is steady and increasing significantly ${ }^{(22)}$. Considering other food groups, our results are in line with other studies, despite the higher availability of cereals and fish/seafood among elderly Portuguese households ${ }^{(21,24,36,41)}$. Compared with other European countries, Portugal reports a reduction in the household availability of most food items ${ }^{(21,36)}$. Our findings for elderly households revealed a decrease in total energy over time that is just accompanied by a reduction in some food items.

Our results showed an increase in the availability of fruits. Higher availability of fruits was also found in Southern European countries in comparison with Northern European countries. A study by Naska et al. ${ }^{(42)}$ using HBS data to assess fruit and vegetable availability patterns in ten European countries reported similar findings. However, the differences in fruit and vegetable consumption that were previously identified between Mediterranean and Northern European countries seem to be levelling out $^{(43)}$.

We found that elderly female individuals living alone had increased availability of vegetables, and this group's availability of fruits and vegetables in 2005 was the highest of all elderly household types. Sex differences in fruit and vegetable intake among older adults were also reported in a study by Baker and Wardle ${ }^{(44)}$. Their results showed that women ate significantly more fruits and vegetables than men and that men's poorer nutrition knowledge could 
Table 3 Daily per capita energy and nutrient availability in elderly Portuguese households. Household Budget Surveys from 1990 to 2005

\begin{tabular}{|c|c|c|c|c|c|c|c|c|c|c|c|c|c|c|c|c|c|c|}
\hline \multirow[b]{3}{*}{ Energy and nutrient } & \multicolumn{12}{|c|}{ Household type } & \multicolumn{6}{|c|}{ Effects $\dagger$} \\
\hline & \multicolumn{4}{|c|}{ Solitary elderly female } & \multicolumn{4}{|c|}{ Solitary elderly male } & \multicolumn{4}{|c|}{ Couple $^{*}$} & \multicolumn{2}{|c|}{ Household type } & \multicolumn{2}{|c|}{ Year } & \multicolumn{2}{|c|}{ Interaction‡ } \\
\hline & 1990 & 1995 & 2000 & 2005 & 1990 & 1995 & 2000 & 2005 & 1990 & 1995 & 2000 & 2005 & $\eta^{2}$ & $P$ & $\eta^{2}$ & $P$ & $\eta^{2}$ & $P$ \\
\hline \multicolumn{19}{|c|}{ Energy (MJ/person per d)§ } \\
\hline Mean & 14.23 & 11.96 & 12.77 & $10 \cdot 56$ & 15.49 & $12 \cdot 64$ & $10 \cdot 16$ & 9.37 & 13.87 & 11.44 & 11.70 & $10 \cdot 49$ & 0.014 & $<0.001$ & 0.014 & $<0.001$ & 0.006 & $<0.001$ \\
\hline SE & 0.37 & 0.31 & 0.35 & 0.31 & 0.97 & 0.74 & 0.54 & 0.57 & 0.20 & 0.14 & 0.18 & 0.16 & & & & & & \\
\hline P25 & $6 \cdot 88$ & $6 \cdot 27$ & $5 \cdot 69$ & 4.90 & $6 \cdot 61$ & 6.58 & 4.10 & 3.11 & 8.52 & 7.04 & 6.85 & 5.76 & & & & & & \\
\hline $\begin{array}{l}\text { Median } \\
\text { P } 75\end{array}$ & $\begin{array}{l}12.33 \\
19.42\end{array}$ & $\begin{array}{l}10 \cdot 38 \\
15.27\end{array}$ & $\begin{array}{l}10.45 \\
16.01\end{array}$ & $\begin{array}{r}8.57 \\
13.89\end{array}$ & $\begin{array}{l}11.27 \\
19.47\end{array}$ & $\begin{array}{l}10.79 \\
15.44\end{array}$ & $\begin{array}{r}8 \cdot 01 \\
14.71\end{array}$ & $\begin{array}{r}7.25 \\
12.11\end{array}$ & $\begin{array}{l}12.49 \\
116.57\end{array}$ & $\begin{array}{r}9.87 \\
14.08\end{array}$ & $\begin{array}{r}9.9 \\
14.38\end{array}$ & $\begin{array}{r}9.06 \\
13.44\end{array}$ & & & & & & \\
\hline \multicolumn{19}{|c|}{ Proteins (\% of total energy)§ } \\
\hline Mean & $\begin{array}{r}14.3 \\
0.3\end{array}$ & $\begin{array}{r}15.0 \\
0.2\end{array}$ & $\begin{array}{r}15.5 \\
0.2\end{array}$ & $\begin{array}{r}17 \cdot 7 \\
0.3\end{array}$ & $\begin{array}{r}12 \cdot 2 \\
0.4\end{array}$ & $\begin{array}{r}14.6 \\
0.4\end{array}$ & $\begin{array}{r}16.0 \\
0.4\end{array}$ & $\begin{array}{r}18 \cdot 8 \\
0.7\end{array}$ & $\begin{array}{r}14.3 \\
0.1\end{array}$ & $\begin{array}{r}15.4 \\
0.1\end{array}$ & $\begin{array}{r}16 \cdot 1 \\
0.2\end{array}$ & $\begin{array}{r}18.5 \\
0.2\end{array}$ & 0.004 & $<0.001$ & 0.027 & $<0.001$ & 0.004 & $<0.001$ \\
\hline $\begin{array}{l}\text { SE } \\
\text { P25 }\end{array}$ & $\begin{array}{l}0.3 \\
9.3\end{array}$ & $\begin{array}{r}0.2 \\
11.2\end{array}$ & $\begin{array}{r}0.2 \\
11.0\end{array}$ & $\begin{array}{r}0.3 \\
12.3\end{array}$ & $\begin{array}{l}0.4 \\
7.9\end{array}$ & $\begin{array}{r}0.4 \\
11.4\end{array}$ & $\begin{array}{r}0.4 \\
11.7\end{array}$ & $\begin{array}{r}0.7 \\
11.8\end{array}$ & $\begin{array}{r}0.7 \\
10.4\end{array}$ & $\begin{array}{l}0.7 \\
11.8\end{array}$ & $\begin{array}{r}0.2 \\
11.9\end{array}$ & 0.2 & & & & & & \\
\hline Median & $13 \cdot 1$ & 14.0 & 14.8 & 16.1 & $12 \cdot 1$ & 14.0 & 14.4 & 16.0 & 13.4 & 14.4 & 14.9 & 17.1 & & & & & & \\
\hline & $17 \cdot 8$ & $17 \cdot 7$ & $18 \cdot 6$ & $21 \cdot 1$ & $15 \cdot 8$ & 18.1 & $19 \cdot 0$ & 22.5 & $17 \cdot 2$ & $18 \cdot 4$ & $18 \cdot 6$ & $22 \cdot 3$ & & & & & & \\
\hline \multicolumn{19}{|c|}{ Lipids (\% of total energy)§ } \\
\hline $\begin{array}{l}\text { Mean } \\
\text { SE }\end{array}$ & $\begin{array}{r}0.0 \\
0.7\end{array}$ & $\begin{array}{r}33.3 \\
0.6\end{array}$ & $\begin{array}{r}32.0 \\
0.5\end{array}$ & $\begin{array}{rl}34.6 & 0.6 \\
0.6\end{array}$ & $\begin{array}{r}26.0 \\
1.4\end{array}$ & $\begin{array}{r}29 \cdot 6 \\
1.1\end{array}$ & $\begin{array}{r}28.2 \\
1.0\end{array}$ & $\begin{array}{r}34.0 \\
1.2\end{array}$ & $\begin{array}{r}33.1 \\
0.4\end{array}$ & $\begin{array}{r}r 4 \cdot 6 \\
0.3\end{array}$ & $\begin{array}{r}32.6 \\
0.3\end{array}$ & $\begin{array}{r}35.8 \\
0.4\end{array}$ & 0.017 & $<0.001$ & 0.008 & $<0.001$ & 0.003 & $<0.001$ \\
\hline P25 & 16.7 & 21.0 & 18.8 & $21 \cdot 1$ & 8.0 & 16.3 & 12.9 & 19.9 & $20 \cdot 3$ & 24.0 & 21.1 & 24.0 & & & & & & \\
\hline $\begin{array}{l}\text { Median } \\
\text { P75 }\end{array}$ & 31.2 & $32 \cdot 8$ & 31.4 & 32.8 & $20 \cdot 8$ & 28.9 & $26 \cdot 9$ & 31.2 & 32.8 & 34.3 & 32.3 & 34.3 & & & & & & \\
\hline \multirow{2}{*}{\multicolumn{19}{|c|}{ Cholesterol (mg)§ }} \\
\hline & & & & & & & & & & & & & & & & & & \\
\hline SE & 8.7 & 8.7 & $\begin{array}{r}2<2.0 \\
7.9\end{array}$ & $\begin{array}{l}200 \cdot 9 \\
9.6\end{array}$ & $\begin{array}{r}100.2 \\
18 \cdot 2\end{array}$ & $\begin{array}{r}195.0 \\
14.4\end{array}$ & 11.7 & $\begin{array}{r}190.0 \\
19.7\end{array}$ & 3.7 & $\begin{array}{l}3.0 \\
3.5\end{array}$ & 3.5 & $\begin{array}{r}200.0 \\
4.7\end{array}$ & 0.000 & $<0.001$ & 0.000 & $<0.001$ & 0.004 & $<0.001$ \\
\hline P25 & 66.8 & $80 \cdot 3$ & $71 \cdot 1$ & 75.5 & 14.0 & 49.2 & 31.7 & 32.9 & 99.2 & 96.1 & 93.8 & $106 \cdot 1$ & & & & & & \\
\hline Median & 174.6 & 162.5 & 154.5 & $161 \cdot 0$ & 78.6 & $140 \cdot 1$ & 11.3 & 131.6 & $184 \cdot 2$ & $173 \cdot 0$ & $170 \cdot 2$ & 193.7 & & & & & & \\
\hline \multirow{2}{*}{\multicolumn{19}{|c|}{ MUFA (\% of total energy)§ }} \\
\hline & & & & & & & & & & & & & & & & & & \\
\hline $\begin{array}{l}\text { Mean } \\
\text { SE }\end{array}$ & $\begin{array}{r}13.5 \\
0.4\end{array}$ & & $\begin{array}{r}13.1 \\
0.3\end{array}$ & $\begin{array}{r}13.5 \\
0.4\end{array}$ & $\begin{array}{r}11.3 \\
0.9\end{array}$ & $\begin{array}{r}12.7 \\
0.7\end{array}$ & $\begin{array}{r}11.3 \\
0.6\end{array}$ & $\begin{array}{r}12.8 \\
0.7\end{array}$ & $\begin{array}{r}14.2 \\
0.2\end{array}$ & $\begin{array}{r}14.8 \\
0.2\end{array}$ & $\begin{array}{r}13.8 \\
0.2\end{array}$ & $\begin{array}{r}14.7 \\
0.2\end{array}$ & 0.021 & $<0.001$ & 0.007 & $<0.001$ & 0.004 & $<0.001$ \\
\hline $\begin{array}{l}\text { SE } \\
\text { P25 }\end{array}$ & $\begin{array}{l}0.4 \\
4.4\end{array}$ & 6.3 & 5.6 & $\begin{array}{l}0.4 \\
6.1\end{array}$ & 1.7 & 4.9 & 3.8 & 5.0 & 6.4 & 7.8 & 6.7 & 7.3 & & & & & & \\
\hline Median & 9.5 & $12 \cdot 1$ & $11 \cdot 0$ & 10.5 & 6.5 & $11 \cdot 0$ & 9.5 & 9.0 & $12 \cdot 9$ & 14.3 & 12.5 & 12.3 & & & & & & \\
\hline \multirow{2}{*}{\multicolumn{19}{|c|}{ SFA (\% of total energy)§ }} \\
\hline & & & & & & & & & & & & & & & & & & \\
\hline $\begin{array}{l}\text { Mean } \\
\text { SE }\end{array}$ & $\begin{array}{l}7.7 \\
0.2\end{array}$ & $\begin{array}{l}8.0 \\
0.1\end{array}$ & $\begin{array}{l}8.0 \\
0.1\end{array}$ & $\begin{array}{r}10.5 \\
0.2\end{array}$ & $\begin{array}{l}5.7 \\
0.4\end{array}$ & $\begin{array}{l}7.2 \\
0.3\end{array}$ & $\begin{array}{l}7.5 \\
0.3\end{array}$ & $\begin{array}{r}10.5 \\
0.4\end{array}$ & $\begin{array}{l}7.3 \\
0.1\end{array}$ & $\begin{array}{l}7.7 \\
0.1\end{array}$ & $\begin{array}{l}7.6 \\
0.1\end{array}$ & $\begin{array}{r}10.0 \\
0.1\end{array}$ & 0.016 & $<0.001$ & 0.031 & $<0.001$ & 0.007 & $<0.001$ \\
\hline $\begin{array}{l}\text { SE } \\
\text { P25 }\end{array}$ & $4 \cdot 6$ & 5.4 & 4.9 & 6.4 & 1.2 & 4.0 & 3.4 & 6.2 & 4.9 & 5.4 & 5.2 & 7.1 & & & & & & \\
\hline $\begin{array}{l}\text { Median } \\
\text { P75 }\end{array}$ & $\begin{array}{r}7.5 \\
10.4\end{array}$ & $\begin{array}{r}7.9 \\
10.3\end{array}$ & $7 \cdot 6$ & $9 \cdot 9$ & 4.5 & $6 \cdot 3$ & $7 \cdot 2$ & 9.8 & $7 \cdot 3$ & $7 \cdot 5$ & $7 \cdot 6$ & 9.8 & & & & & & \\
\hline \multicolumn{19}{|c|}{ PUFA (\% of total energy)§ } \\
\hline Mean & $7 \cdot 3$ & 7.3 & 6.4 & 6.5 & 5.4 & 5.9 & $5 \cdot 3$ & $6 \cdot 6$ & 7.5 & 8.0 & 6.8 & 7.1 & 0.018 & $<0.001$ & 0.004 & $<0.001$ & 0.000 & 0.585 \\
\hline SE & 0.3 & 0.2 & 0.2 & 0.2 & 0.4 & 0.3 & 0.3 & 0.4 & 0.1 & 0.1 & 0.1 & 0.1 & & & & & & \\
\hline P25 & $2 \cdot 0$ & 2.9 & $2 \cdot 3$ & $2 \cdot 8$ & 1.8 & $2 \cdot 4$ & $2 \cdot 1$ & $2 \cdot 4$ & 2.7 & 3.8 & $2 \cdot 9$ & 3.1 & & & & & & \\
\hline Median & 3.8 & $5 \cdot 2$ & 4.0 & 4.1 & 2.9 & 3.8 & 3.3 & 3.9 & 4.5 & $7 \cdot 3$ & $5 \cdot 1$ & $5 \cdot 2$ & & & & & & \\
\hline $\begin{array}{l}\text { P75 } \\
\text { Carbohydrates (\% of }\end{array}$ & $12 \cdot 2$ & 11.4 & 9.8 & 7.9 & $5 \cdot 9$ & $9 \cdot 3$ & 8.2 & 7.9 & $11 \cdot 7$ & $11 \cdot 3$ & 9.8 & $10 \cdot 4$ & & & & & & \\
\hline $\begin{array}{l}\text { Carbohydrates (\% of } \\
\text { Mean }\end{array}$ & 55.3 & $54 \cdot 2$ & 55.6 & $49 \cdot 8$ & 53.2 & $52 \cdot 3$ & 53.7 & 46.6 & $51 \cdot 4$ & 49.5 & $51 \cdot 2$ & 45.5 & 0.006 & $<0.001$ & 0.009 & $<0.001$ & 0.001 & 0.134 \\
\hline & 0.7 & 0.6 & 0.5 & 0.6 & 1.5 & 1.2 & 1.2 & 1.3 & 0.3 & 0.3 & 0.3 & 0.4 & & & & & & \\
\hline P25 & 41.4 & 43.4 & 44.5 & 37.9 & 37.5 & $40 \cdot 0$ & 38.8 & 32.3 & $41 \cdot 6$ & 40.8 & 40.9 & 34.5 & & & & & & \\
\hline Median & $55 \cdot 3$ & $53 \cdot 6$ & 54.1 & 50.7 & 54.4 & 52.1 & 53.9 & 43.5 & 50.6 & $49 \cdot 3$ & 50.6 & 45.5 & & & & & & \\
\hline & 68.5 & 64.0 & $66 \cdot 6$ & 62.5 & $69 \cdot 2$ & $62 \cdot 7$ & 67.0 & $61 \cdot 6$ & $61 \cdot 2$ & $57 \cdot 9$ & 60.6 & 56.7 & & & & & & \\
\hline $\begin{array}{l}\text { Simple sugars }(\% \text { of } \\
\text { Mean }\end{array}$ & & & & & & & & & & & & & & & & $<0.001$ & 0.003 & $<0.001$ \\
\hline $\begin{array}{l}\text { Mean } \\
\text { SE }\end{array}$ & $\begin{array}{r}22.5 \\
0.6\end{array}$ & $\begin{array}{r}18.7 \\
0.4\end{array}$ & $\begin{array}{r}21.4 \\
0.4\end{array}$ & $\begin{array}{r}17.8 \\
0.4\end{array}$ & $\begin{array}{r}15.9 \\
1.1\end{array}$ & $\begin{array}{r}16.0 \\
0.9\end{array}$ & $\begin{array}{r}18.1 \\
1.0\end{array}$ & $\begin{array}{r}17.3 \\
0.9\end{array}$ & $\begin{array}{r}17.5 \\
0.2\end{array}$ & $\begin{array}{r}15.3 \\
0.2\end{array}$ & $\begin{array}{r}17.3 \\
0.2\end{array}$ & $\begin{array}{r}15.5 \\
0.2\end{array}$ & 0.022 & $<0.001$ & 0.003 & $<0.001$ & 0.003 & $<0.001$ \\
\hline P25 & 11.0 & 11.0 & $12 \cdot 6$ & 9.0 & 4.1 & 7.4 & 8.4 & 7.0 & 10.4 & 9.5 & 10.8 & 8.6 & & & & & & \\
\hline Median & 19.9 & $16 \cdot 9$ & $18 \cdot 7$ & $15 \cdot 3$ & 12.4 & 13.2 & $14 \cdot 3$ & 14.4 & 16.4 & 13.9 & 15.8 & 13.3 & & & & & & \\
\hline P75 & $29 \cdot 3$ & 24.0 & $27 \cdot 2$ & $23 \cdot 1$ & $24 \cdot 2$ & 20.8 & $23 \cdot 5$ & $22 \cdot 1$ & 22.9 & 19.5 & $21 \cdot 7$ & 19.9 & & & & & & \\
\hline
\end{tabular}


explain a significant part of their lower intake of these foods. This situation may also be related to elderly females' concern with nutrition and healthy choices, aspects noted by a study on the determinants of food choice and the food habits of elderly populations that included Portuguese individuals ${ }^{(45)}$. Alternatively, living alone may cause a loss of interest and ability in cooking, leading to the substitution of meals by more convenient food items, such as milk/milk products and fruits. Furthermore, the sociodemographic characteristics of these households may be linked to household food availability. For example, the couple elderly households showed the highest availability per capita for nine of the fourteen food and beverage items analysed. Additionally, the presence of a woman and/or another person, such as in the couple elderly households, may play an important role in household dietary availability, as described by Morais ${ }^{(45)}$. Our results regarding higher availability of food and beverage groups in couple and solitary elderly female household types may indicate this phenomenon. Moreover, over the years, the elderly households were increasingly located in urbanized areas, which impose a different lifestyle that may have contributed to differing food availability within these households. The higher median values of the eating out expenses of solitary elderly male households may be related to this situation. Similar results were presented by Morais ${ }^{(45)}$.

Over time, the availability of alcoholic beverages decreased within elderly households, but solitary elderly males had the highest mean values for the availability of these items. Other studies using direct measures of intake have reported that men have higher ethanol intake ${ }^{(46)}$ and alcohol intake than woman ${ }^{(16,47,48)}$. A study by Vaz de Almeida et $a .^{(49)}$ produced similar findings. Their investigation of the social and cultural aspects of alcohol consumption in a sample of older people in eight European countries revealed that alcohol consumption was connected with culture and that it had social and psychological implications. Their results also suggested that alcohol intake in Southern European countries is a long-established and entrenched social behaviour that is related to male preferences and male conviviality. We also need to consider that the substantial variation over time in some of the analysed food groups may have to do with differences in the food group definition; mistake or genuine disparity can also be highlighted as some of the plausible explanations for our findings.

Considering overall household food availability, the higher food availability of the elderly may reflect less frequent eating out ${ }^{(50)}$ and/or a tendency to accumulate food in the household. Over time, other studies have reported the decrease of in-household dietary availability $^{(39)}$ which is certainly due to the increase of eating out of home, for which expenses have increased significantly in populations ${ }^{(50)}$, as was also found in the present study results. Our results show that the energy availability and macronutrient contribution to the total energy available in elderly households changed over time. The availability of total energy and the proportion of carbohydrates decreased over time, whereas the proportions of proteins and SFA increased. Similar results were found for other European countries ${ }^{(43)}$. However, we found that the solitary elderly female household type experienced a decrease in the proportion of lipids, which may be related to female health concerns, as highlighted by other studies ${ }^{(47)}$.

Few studies have analysed elderly dietary data in Portugal. Despite methodological differences ${ }^{(27,46,51)}$, some comparisons can be performed. In Epi-Porto ${ }^{(46)}$ macronutrient adequacy was analysed, showing that older individuals consumed less than $20 \%$ of their total energy from fat. Men aged 70 years and older presented the lowest inadequacy proportion of carbohydrate intake. Marques-Vidal et al. ${ }^{(51)}$ studied representative samples of the Portuguese population and found that, in general, the consumption of traditional foods such as fish and soup declined, but in a smaller proportion among elderly people. They also commented that at the individual level, higher consumption of meat and milk would contribute to higher relative protein and fat intakes.

Comparing our results with the population's nutrient intake goals ${ }^{(4)}$, we found that the median values for the proportions of protein and lipids exceeded the recommendations, and the median values for carbohydrate proportion were below the recommendations. Rodrigues et $a l^{(30)}$ analysed the availability of food, energy and nutrients for elderly people living alone. Their results showed that the availability of sugar and sugar products was above the median for the total population. Their most relevant findings were the low availability of energy derived from carbohydrates and high values for total dietary energy, protein and fats.

Another study by Rodrigues et al. ${ }^{(23)}$ revealed that Portuguese solitary elderly households and households with two elderly members presented low levels of compliance with the WHO dietary goals. Furthermore, another study reported low dietary quality levels for European elders ${ }^{(29)}$. This therefore highlights that sociodemographic differences in dietary profile should be considered when designing health and nutrition initiatives.

Portuguese household food availability data from HBS have been analysed within the DAFNE initiative since the 1990s, contributing to information about the country's dietary patterns ${ }^{(19,24,39)}$. The HBS are surveys that are regularly conducted at the country level with information based on descriptions of the food entering the household (identification of the food quantity acquired and the amount of money spent). Despite various limitations, the HBS have been used to describe food availability in Portugal, and they represent an important source of nationally representative dietary information. These characteristics can be considered strengths of our study. 
Nevertheless, the HBS also present some drawbacks. For instance, the fact that one cannot determine the fraction of food acquired that was not consumed (e.g. loss, waste, fed to pets), the non-specification of the consumption of foods and beverages outside the household, and the inability to estimate the real distribution of food among household members can be considered some of the limitations of these sources of information in the characterization of diets. Sampling process issues, the sampling period adopted as a reference for the calculation of food supplies, and limitations to the process of collecting, recording and interpreting the data also deserve special consideration when reporting and analysing the results of these studies $^{(52-54)}$. In general, the different time periods of data collection (7 d in 1990 and $14 \mathrm{~d}$ from 1995 onwards) used in the analysed surveys is not expected to impair comparisons $^{(43)}$. However, it may have an impact on the mean values of the rarely consumed foods/beverages. Another important issue is the fact that elderly Portuguese individuals in urban and rural areas have coverage of home care services, including the provision of meals, which may affect measures of household food availability by HBS.

The lack of detailed information about food items acquired outside the household is a limitation when using HBS food, beverage and derived nutrient data. However, it is important to note that households with older persons generally present fewer food expenses outside the household $^{(45,50)}$.

The results presented in the current paper reveal differences in household food availability among elderly household types. The increase in the availability of milk/ milk products and non-alcoholic beverages, together with the decrease in cereals and potatoes, suggest that food items are being replaced over time, turning this population's food pattern into one more closely associated with the so-called 'modern' diet ${ }^{(55)}$. A healthier trend was observed in the global decreasing availability of sugar/ sugar products and in the increasing availability of fruits and vegetables and the decreasing availability of alcoholic beverages for solitary elderly female and couple households, as also noted by Rodrigues et al. ${ }^{(39)}$ for the entire population.

Given the need to make decisions about nutritional and health policies designed for older persons, our findings provide recommendations to achieve healthier dietary availability among elderly households. Our results encourage the increased availability of traditional foods, such as pulses and fish, which are components of the Mediterranean diet and are part of the Portuguese cultural heritage. These recommendations may help to improve dietary quality by contributing to a reduction in lipid proportions and an increase in the carbohydrate proportion.

The demographic changes characterized by the increase of the elderly population have occurred in conjunction with changes in habits, including the characteristics of dietary availability in households. Further research using direct measures of food intake and regular dietary monitoring are needed to clarify the dietary habits of older people and their association with sociodemographic characteristics. The use of HBS dietary data should be expanded and improved by exploring different household compositions to improve knowledge about elderly groups. This research would contribute to revealing disparities and would constitute a basis for developing national and regional nutrition policies aimed at increasing the foodrelated health and well-being of this specific age group, especially in light of the need to incorporate a life course approach $^{(11)}$. Combining HBS dietary data with direct measures of food intake could further clarify the dietary habits of older people and their association with sociodemographic characteristics.

\section{Acknowledgements}

Financial support: D.M.S. is a PhD student with scholarship supported by the Fundação para Ciência e Tecnologia (FCT grant number SFRH/BD/62734/2009), Portugal and by the University of the State of Rio de Janeiro Professor Training Program (PROCAD/SR2 5096/2009), Brazil. The Fundação para Ciência e Tecnologia and University of the State of Rio de Janeiro Professor Training Program had no role in the design, analysis or writing of this article. Conflict of interest: None. Authorship: D.M.S. conceived and executed the project supervised by the other authors. The data set was harmonized by S.S.P.R. and D.M.S. D.M.S. and B.M.P.M.O. performed the statistical analyses and interpreted the results. The paper was drafted by D.M.S. in consultation with S.S.P.R., B.M.P.M.O. and M.D.V.d.A. All authors revised the paper. Ethics of buman subject participation: Ethical approval was not required. Consent to use the Portuguese HBS data according to data protection and confidentiality protocols was requested from the National Institute of Statistics.

\section{References}

1. European Commission (2007) Healthy Ageing: Keystone for a Sustainable Europe. Geneva: Health \& Consumer Protection Directorate-General, European Commission.

2. European Commission (2011) Active Ageing and Solidarity between Generations: A Statistical Portrait of the European Union 2012. Luxembourg: Eurostat.

3. Gonçalves C \& Carrilho MJ (2006) Envelhecimento crescente mas espacialmente desigual. Revista de Estudos Demográficos no. 40, pp. 22-37. Lisboa: INE.

4. World Health Organization (2003) Diet, Nutrition, and the Prevention of Chronic Diseases. Joint WHO/FAO Expert Consultation. WHO Technical Report Series no. 916. Geneva: WHO.

5. World Health Organization (2009) Global Health Risks Mortality and Burden of Disease Attributable to Selected Major Risks. Geneva: WHO; available at http://www.who. int/healthinfo/global_burden_disease/global_health_risks/ en/index.html 
6. Inzitari I, Doets E, Bartali B et al. (2011) Nutrition in the agerelated disablement process. J Nutr Health Aging 15, 599-604.

7. Phillips F (2003) Nutrition for healthy ageing. Nutr Bull 28, 253-263.

8. Hu FB (2002) Dietary pattern analysis: a new direction in nutritional epidemiology. Curr Opin Lipidol 13, 3-9.

9. Kourlaba G \& Panagiotakos DB (2009) Dietary quality indices and human health: a review. Maturitas 62, 1-8.

10. Huijbregts P, Feskens E, Räsänen L et al. (1997) Dietary pattern and 20 year mortality in elderly men in Finland, Italy, and the Netherlands: longitudinal cohort study. BMJ 315, 13-17.

11. Ben-Shlomo Y \& Kuh D (2002) A life course approach to chronic disease epidemiology: conceptual models, empirical challenges and interdisciplinary perspectives. Int J Epidemiol 31, 285-293.

12. Ferreira FAG \& Cruz JAA (1985) Inquérito Alimentar Nacional ( $1^{\mathrm{a}}$ Parte). Revista do Centro de Estudos de Nutrição do Instituto Nacional de Saúde Dr. Ricardo Jorge 9, issue 4. Lisboa: INSA.

13. Ferreira FAG \& Cruz JAA (1986) Inquérito Alimentar Nacional ( $2^{\mathrm{a}}$ Parte). Revista do Centro de Estudos de Nutrição do Instituto Nacional de Saúde Dr. Ricardo Jorge 10 , issue 2-3. Lisboa: INSA.

14. Ferreira FAG \& Cruz JAA (1987) Inquérito Alimentar Nacional ( $3^{\text {a }}$ Parte). Revista do Centro de Estudos de Nutrição do Instituto Nacional de Saúde Dr. Ricardo Jorge 11 , issue 3. Lisboa: INSA.

15. Poínhos R, Franchini B, Afonso C et al. (2009) Alimentação e estilos de vida da população portuguesa: metodologia e resultados preliminares. Alimentação Humana 15, 43-60.

16. Instituto Nacional de Estatística \& Instituto Nacional de Saúde Dr. Ricardo Jorge (2009) Inquérito Nacional de Saúde 2005/2006. Lisboa: INE and INSA.

17. Lagiou P, Trichopoulou A \& Dafne Contributors (2001) The DAFNE initiative: the methodology for assessing dietary patterns across Europe using household budget survey data. Public Health Nutr 4, 1135-1141.

18. European Commission, Health \& Consumer Protection Directorate-General (2005) The DAFNE Food Classification System. Operationalisation in 16 European Countries. Luxembourg: Services of the European Commission.

19. Rodrigues SSP \& de Almeida MD (2001) Portuguese household food availability in 1990 and 1995. Public Health Nutr 4, 1167-1171.

20. Trichopoulou A, Naska A \& Oikonomou E (2005) The DAFNE databank: the past and future of monitoring the dietary habits of Europeans. J Public Health 13, 69-73.

21. Trichopoulou A, Naska A, Costacou T et al. (2002) Disparities in food habits across Europe. Proc Nutr Soc 61, 553-558.

22. Naska A, Bountziouka V \& Trichopoulou A (2010) Soft drinks: time trends and correlates in twenty-four European countries. A cross-national study using the DAFNE (Data Food Networking) databank. Public Health Nutr 13, $1346-1355$.

23. Rodrigues SSP, Caraher M, Trichopoulou A et al. (2007) Portuguese households' diet quality (adherence to Mediterranean food pattern and compliance with WHO population dietary goals): trends, regional disparities and socioeconomic determinants. Eur J Clin Nutr 62, $1263-1272$

24. Rodrigues SSP, Trichopoulou A \& De Almeida MDV (2008) Household diet quality in relation to mortality in Portuguese regions: an ecological study. J Public Health 16, 43-51.

25. Rodrigues SSP, Lopes C, Naska A et al. (2007) Comparison of national food supply, household food availability and individual food consumption data in Portugal. $J$ Public Health 15, 447-455.
26. Naska A, Berg MA, Cuadrado C et al. (2009) Food balance sheet and household budget survey dietary data and mortality patterns in Europe. BrJ Nutr 102, 166-171.

27. Volkert D (2005) Nutrition and lifestyle of the elderly in Europe. J Public Health 13, 56-61.

28. Afonso CIPN (2012) Dietary habits and body weight in aging: a study in elderly Europeans. PhD Thesis, University of Porto (in Portuguese)..

29. Irz X, Fratiglioni L, Kuosmanen $\mathrm{N}$ et al. (2013) Sociodemographic determinants of diet quality of the EU elderly: a comparative analysis in four countries. Public Health Nutr (Epublication ahead of print version).

30. Rodrigues SSP \& Vaz de Almeida MD (2006) Disponibilidade de alimentos, energia e nutrientes em idosos Portugueses que vivem sozinhos. Alimentação Humana 12, 39-44.

31. Instituto Nacional de Estatística (1990) Inquérito aos Orçamentos Familiares 1989-1990. Metodologia. Lisboa: INE.

32. Instituto Nacional de Estatística (1997) Inquérito aos Orçamentos Familiares 1994/95. Metodologia. Lisboa: INE.

33. Instituto Nacional de Estatística (2002) Inquérito aos Orçamentos Familiares 2000. Metodologia. Lisboa: INE.

34. Instituto Nacional de Estatística (2005) Documento Metodológico: IDF - Inquéritos ás Despesas das Famílias. Lisboa: INE.

35. Instituto Nacional de Estatística (2002) O Envelhecimento em Portugal: Situação Demográfica e Sócio-Económica Recente das Pessoas Idosas. Lisboa: INE.

36. Rodrigues SSP, Naska A, Trichopoulou A et al. (2007) Availability of foods and beverages in nationally representative samples of Portuguese households from 1990 to 2000: the DAFNE initiative. J Public Health 15, 211-220.

37. Martins I, Porto A \& Oliveira L (2010) Tabela da Composição de Alimentos. Lisboa: INSA.

38. Cohen J (1988) Statistical Power Analysis for the Behavioral Sciences. Hillsdale, NJ: Lawrence Erlbaum Associates.

39. Rodrigues S, Rowcliffe P \& de Almeida MDV (2010) Evolução da Disponibilidade de Alimentos e Bebidas em Portugal - Projecto ANEMOS. Porto: Faculdade de Ciências da Nutrição e Alimentação, Universidade do Porto; available at http://sigarra.up.pt/ant/fcnaup_uk/PUBLS_PESQUISA. FORMVIEW?P ID $=8584$

40. Chen Q \& Marques-Vidal P (2007) Trends in food availability in Portugal in 1966-2003. Eur J Nutr 46, 418-427.

41. Trichopoulou A, Henderickx HK, Remaut-de Winter AM et al. (2001) The DAFNE databank as a simple tool for nutrition policy. Public Health Nutr 4, 1187-1198.

42. Naska A, Vasdekis VGS, Trichopoulou A et al. (2000) Fruit and vegetable availability among ten European countries: how does it compare with the 'five-a-day' recommendation? Br J Nutr 84, 549-556.

43. Naska A, Fouskakis D, Oikonomou E et al. (2006) Dietary patterns and their socio-demographic determinants in 10 European countries: data from the DAFNE databank. Eur J Clin Nutr 60, 181-190.

44. Baker AH \& Wardle J (2003) Sex differences in fruit and vegetable intake in older adults. Appetite 40, 269-275.

45. Morais CM (2013) Determinants of food choices and food habits of elderly populations. PhD Thesis, University of Porto.

46. Lopes C, Oliveira A, Santos A et al. (2006) Consumo Alimentar no Porto. Porto: Faculdade de Medicina, Universidade do Porto; available at http://higiene.med.up. $\mathrm{pt} /$ consumoalimentarporto

47. Wardle J, Haase AM, Steptoe A et al. (2004) Gender differences in food choice: the contribution of health beliefs and dieting. Ann Behav Med 27, 107-116.

48. Oliveira A, Lopes C, Santos A et al. (2008) Ingestão de macronutrients e de etanol em adultos Portugueses. Acta Med Port 21, 37-48. 
49. Vaz de Almeida MD, Davidson K, De Morais C et al. (2005) Alcohol consumption in elderly people across European countries: results from the Food in Later Life Project. Ageing Int 30, 377-395.

50. Bezerra IN \& Sichieri R (2010) Characteristics and spending on out-of-home eating in Brazil. Rev Saude Publica 44, 221-229.

51. Marques-Vidal P, Ravasco P, Dias C et al. (2006) Trends of food intake in Portugal, 1987-1999: results from the National Health Surveys. Eur J Clin Nutr 60, 1414-1422.
52. Gibson RS (2005) Food consumption at the national and household levels. Principles of Nutritional Assessment, 2nd ed., pp. 27-40. New York: Oxford University Press.

53. World Health Organization (1991) Food and Health Data: Their Use in Nutrition Policy-Making. Copenhagen: WHO Regional Office for Europe.

54. United Nations (2005) Household Sample Surveys in Developing and Transition Countries. New York: UN.

55. Popkin BM (2006) Global nutrition dynamics: the world is shifting rapidly toward a diet linked with noncommunicable diseases. Am J Clin Nutr 84, 289-298. 